\title{
Nucleosynthesis and chemical evolution of intermediate-mass stars: results from planetary nebulae
}

\author{
Walter J. Maciel ${ }^{* \dagger}$ \\ University of São Paulo \\ E-mail:maciel@astro.iag.usp.br
}

Roberto D. D. Costa

University of São Paulo

E-mail: roberto@astro.iag.usp.br

Thais E. P. Idiart

University of São Paulo

E-mail: thais@astro.iag.usp.br

\begin{abstract}
Planetary nebulae (PN) are an excellent laboratory to investigate the nucleosynthesis and chemical evolution of intermediate mass stars. In these objects accurate abundances can be obtained for several chemical elements that are manufactured or contaminated by the PN progenitor stars, such as $\mathrm{He}, \mathrm{N}, \mathrm{C}$, and also elements that were originally produced by more massive stars of previous generations, namely $\mathrm{O}, \mathrm{Ne}, \mathrm{Ar}$, and $\mathrm{S}$. Some of these elements are difficult to study in stars, so that $\mathrm{PN}$ can be used in order to complement results obtained from stellar data.

In the past few years, we have obtained a large sample of PN with accurately derived abundances, including objects of different populations, namely the solar neighbourhood, the galactic disk and anticentre, the galactic bulge and the Magellanic Clouds.

In this work, we present the results of our recent analysis of the chemical abundances of $\mathrm{He}$, $\mathrm{O}, \mathrm{N}, \mathrm{S}, \mathrm{Ar}$ and $\mathrm{Ne}$ in galactic and Magellanic Cloud PN. Average abundances and abundance distributions of all elements are determined, as well as distance-independent correlations. These correlations are particularly important, as they can be directly compared with the predictions of recent theoretical evolutionary models for intermediate mass stars.
\end{abstract}

11th Symposium on Nuclei in the Cosmos

19-23 July, 2010

Heidelberg, Germany.

*Speaker.

${ }^{\dagger}$ Work partially supported by FAPESP/CNPq. 


\section{Introduction}

Planetary nebulae $(\mathrm{PN})$ are an excellent laboratory to investigate the nucleosynthesis and chemical evolution of intermediate mass stars. Accurate abundances can be obtained for several chemical elements, including (i) those elements that are manufactured by the PN intermediate-mass progenitor stars $(\mathrm{He}, \mathrm{N}, \mathrm{C})$, and (ii) also elements that were originally produced by more massive stars of previous generations $(\mathrm{O}, \mathrm{Ne}, \mathrm{Ar}, \mathrm{S})$. The abundances of the first class of elements measured in PN include the original content previous to the formation of the progenitor stars and the contamination effects during the nuclear processes in these objects. As a consequence, $\mathrm{PN}$ can be used to study the nucleosynthetic processes in intermediate mass stars. On the other hand, elements such as $\mathrm{O}, \mathrm{Ne}$, etc. reveal the interstellar abundances at the time and place the progenitor stars were formed, so that the determination of their chemical abundances produces important observational constraints to the chemical evolution models for the galaxies hosting these objects.

In the past few years, we have obtained a large sample of PN of different galactic populations with accurately derived abundances (cf. Maciel et al. [7], [8], and references therein). In this work we present average abundances and abundance distributions of several elements, as well as distance-independent abundance correlations that can be directly compared with the predictions of recent theoretical evolutionary models for intermediate mass stars.

\section{The sample}

For the Milky Way, we have considered three different samples: (i) our own IAG/USP data (cf. Maciel et al. [7], [8]), which includes 84 disk PN and 188 bulge PN, supplemented by 84 bulge nebulae from Stasińska et al. [12]; (ii) Sample A, which includes 234 PN in the Milky Way disk (Maciel et al. [9], Maciel and Costa [6]), and (iii) Sample B, which is a larger sample which includes literature data, presently containing 372 nebulae. For the Magellanic Clouds, we have again considered our own data (Maciel et al. [7]), including 45 nebulae for the SMC and 23 objects in the LMC, supplemented by data from Stasińska et al. [12], with 48 PN in the SMC and 106 in the LMC, and Leisy and Dennefeld [5], with 36 PN in the SMC and 120 in the LMC. As discussed by Maciel et al. [7], the merged sample maintains the homogeneity of the individual samples, in view of the similar methods employed, so that a more comprehensive sample can be obtained.

\section{Average abundances and abundance distributions}

We have determined average abundances of all studied elements both in the Galaxy and in the Magellanic Clouds. The He abundances are similar in all samples within the average uncertainties, and are as follows: $\mathrm{He} / \mathrm{H} \simeq 0.115,0.105$, and 0.095 for the Milky Way, the LMC and the SMC, respectively. Typical uncertainties in the He abundances are approximately 0.020 to 0.030 , and the abundances range from 0.05 to 0.18 (Maciel et al. [8]). The $\mathrm{O} / \mathrm{H}$ abundances, which are usually the best determined of all heavy elements considered here, also show a good agreement among all different samples for a given system. In all cases the LMC is richer than the SMC, as expected, and the average metallicity differences are usually in the range 0.3 to $0.5 \mathrm{dex}$, which is consistent with the metallicities given by Stanghellini [11], namely $Z=0.004$ and $Z=0.008$ for the SMC and 

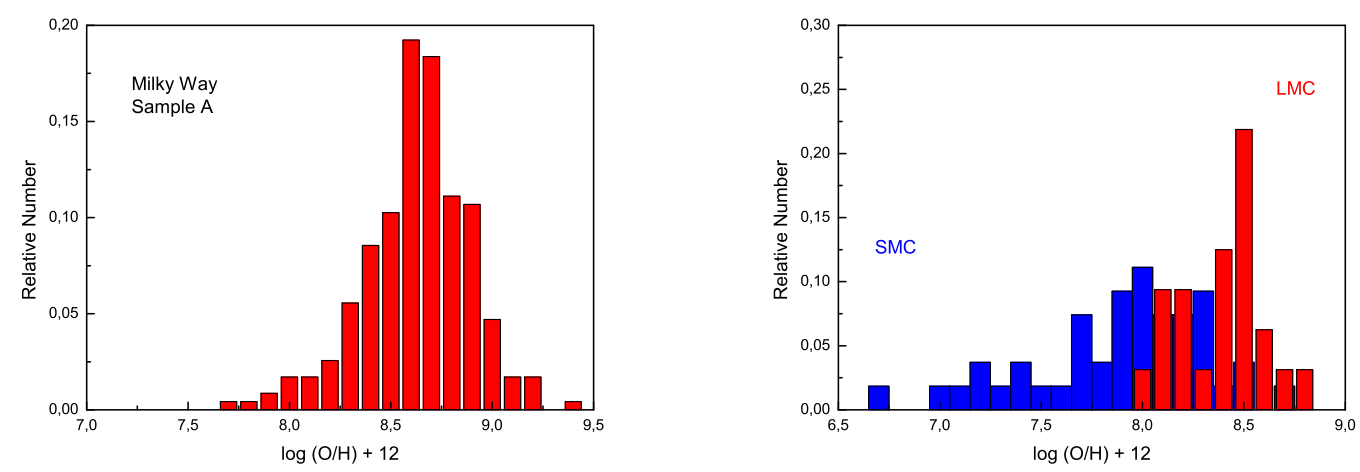

Figure 1: Average oxygen abundances of PN in the Milky Way (left) and Magellanic Clouds (right).

LMC, respectively. The same pattern is observed as the Milky Way data are included, as expected from the larger metallicity of the Galaxy relative to the Magellanic Clouds. The $\mathrm{Ar} / \mathrm{H}$ and $\mathrm{Ne} / \mathrm{H}$ ratios show a behaviour similar to $\mathrm{O} / \mathrm{H}$ in all cases. The sulfur abundances seem to be less reliable, since larger standard deviations are obtained and the average S/H ratio in the Galaxy seems to be lower than in the Magellanic Clouds, contrary to the pattern of the remaining heavy elements. The nitrogen abundances also follow the same pattern as $\mathrm{O} / \mathrm{H}, \mathrm{Ar} / \mathrm{H}$, and $\mathrm{Ne} / \mathrm{H}$. The $\mathrm{N} / \mathrm{H}$ ratio is affected by the dredge-up episodes in the PN progenitor stars, especially the first dredge-up in low mass stars and the second dredge-up in intermediate mass stars, apart from hot bottom burning (HBB) in the more massive progenitors. From our results, the average contamination from the PN progenitor stars is probably small. An example of the metallicity distribution is shown in Figure 1 for the Milky Way and the Magellanic Clouds, where we have considered Sample A and the IAG data, respectively.

\section{Abundance correlations}

The variation of the ratios $\mathrm{Ne} / \mathrm{H}, \mathrm{Ar} / \mathrm{H}$, and $\mathrm{S} / \mathrm{H}$ with $\mathrm{O} / \mathrm{H}$ usually shows a good positive correlation for all systems in the Local Group. This can be seen in Figure 2, where Ne abundances are plotted against the $\mathrm{O} / \mathrm{H}$ ratio for the Milky Way and the Magellanic Clouds. Bulge PN, not shown in the figure, follow the same trend. The correlations are usually very good, with slopes in the range $0.8-1.2$, with the exception of sulfur. As discussed in detail by Maciel et al. [7], there are still problems in the interpretation of sulfur data from PN, a conclusion supported by the comparison of the present results with some recent Spitzer data (Bernard-Salas et al. [1]). It has been suggested ([1], [2]) that the uncertainties in the S/H abundances may be due to the possibility that the adopted ionization correction factors overestimate the contribution of the $\mathrm{S}^{+3}$ ion the total sulfur abundances. Figure 2 also shows the Ne/O ratio, from which we note that this ratio is essentially constant for all metallicities with a relatively small dispersion, in contrast with the elements produced by the progenitor stars, as we will see in the following.

More interesting results can be obtained considering the elements that are produced during the evolution of the PN progenitor stars, namely, $\mathrm{He}, \mathrm{N}$, and $\mathrm{C}$. A plot of the ratio N/H as a function of $\mathrm{O} / \mathrm{H}$ leads to a positive correlation, as in the case of $\mathrm{Ne}$ and $\mathrm{Ar}$, but with a larger dispersion. This is 

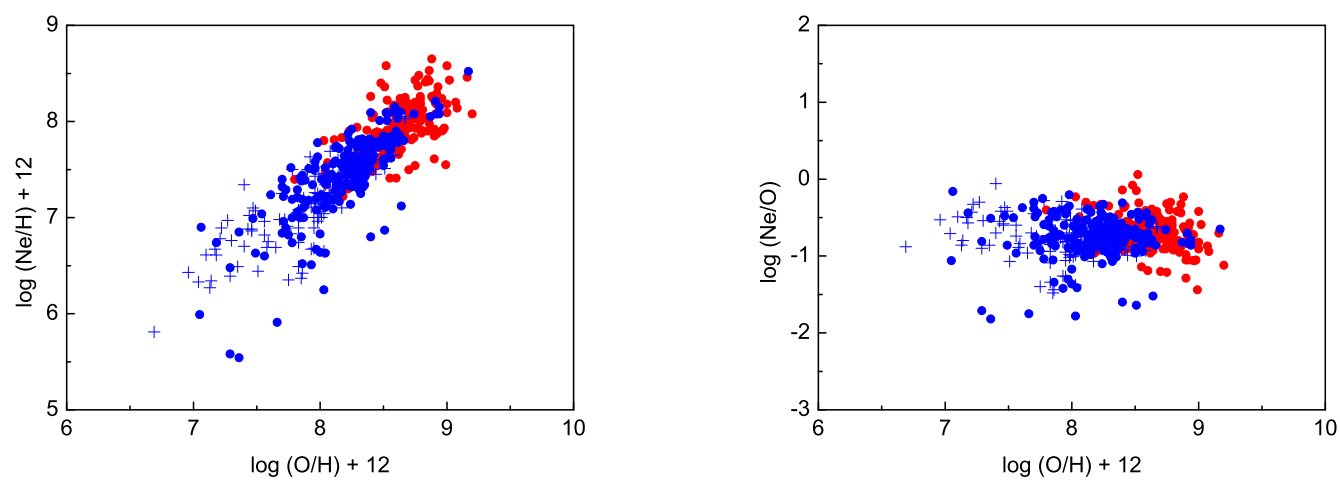

Figure 2: Neon abundances of PN in the Milky Way (red) and Magellanic Clouds (blue).
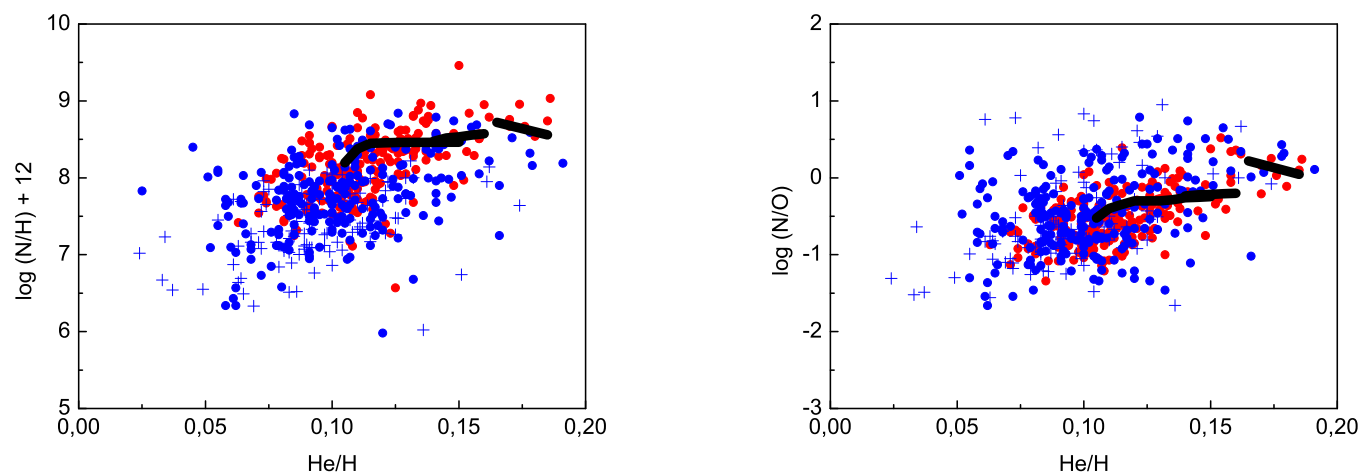

Figure 3: Nitrogen abundances of PN and comparison with theoretical models by Marigo.

due to the fact that the PN display both the original $\mathrm{N}$ present at the formation of the star plus the contamination by the progenitor star. In other words, the N/H ratio measured in PN shows some contamination, or enrichment, in comparison with the original abundances in the progenitor star. The plot of the N/O ratio as a function of $\mathrm{O} / \mathrm{H}$ also shows a larger dispersion in comparison with the Ne/O plot shown in Figure 2, for the same reason.

Very interesting abundance correlations involving $\mathrm{N}$ abundances are those obtained as functions of the He abundances, as shown in Figures 3 and 4. It can be seen that the evolution of the abundances is consistent with a similar trend for all systems, since the slopes are similar for all objects. In figure 3 we have also included the predictions of theoretical models by Marigo et al. [10], shown by the thick lines. These are synthetic evolutionary models for the thermally-pulsing Asymptotic Giant Branch stars (TP-AGB) with masses in the range 1.1 to $5 \mathrm{M}_{\odot}$, in which the first, second and third dredge-up episodes occur, apart from HBB for the most massive objects. These processes affect the $\mathrm{He} / \mathrm{H}$ ratio, and in fact most objects present some enhancement compared to the solar values. According to Marigo et al. [10], progenitors having 0.9 to $4 \mathrm{M}_{\odot}$ and solar composition can explain the "normal" abundances, $\mathrm{He} / \mathrm{H}<0.15$, while for those objects with higher enhancements $(\mathrm{He} / \mathrm{H}>0.15)$ larger masses are needed, in the range 4 to $5 \mathrm{M}_{\odot}$ plus 


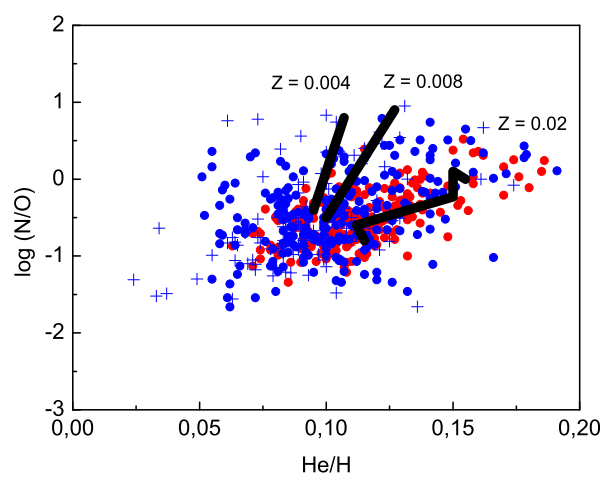

Figure 4: Nitrogen abundances of PN and comparison with theoretical models by Karakas.

an efficient HBB. Similar plots can be obtained using the models developed by Karakas (see for example Karakas and Lattanzio [4]), in which case the initial masses range from 1.0 to $6.0 \mathrm{M}_{\odot}$, and metallicities $Z=0.02, Z=0.008$ and $Z=0.004$ have been considered, as shown in Figure 4 . The agreement for the N/O ratio is good, with a similar conclusion for $\mathrm{N} / \mathrm{H}$, and with a better agreement for the Milky Way, as expected, since this galaxy is somewhat more metal rich than the Magellanic Clouds. We found a continuous transition between the "normal" and He-rich nebulae, which is probably due to the fact that we have a much larger sample compared to Marigo et al. [10]. These results suggest that the nucleosynthetic processes occurring in these systems are similar, even though the global metallicity may be different and the chemical evolution may be affected by different star formation rates. More detailed discussions can be found in some of our recent papers ([3], [7], [8]).

\section{References}

[1] Bernard-Salas, J., Pottasch, S. R., Gutenkunst, S., Morris, P. W., \& Houck, J. R. 2008, ApJ, 672, 274

[2] Henry, R. B., Kwitter, K. B., Balick, B. 2004, AJ, 127, 2284

[3] Idiart, T. P., Maciel, W. J., Costa, R. D. D. 2007, A\&A 472, 101

[4] Karakas, A., Lattanzio, J. C. 2007, PASA 24, 103

[5] Leisy, P., Dennefeld, M. 2006, A\&A 456, 451

[6] Maciel, W. J., Costa, R. D. D. 2010, Asymmetric Planetary Nebulae 5, (in press)

[7] Maciel, W. J., Costa, R. D. D., Idiart, T. E. P. 2009, RMAA 45, 127

[8] Maciel, W. J., Costa, R. D. D., Idiart, T. E. P. 2010, IAU Symp. 268, ed. C. Charbonnel, M. Tosi, F. Primas, C. Chiappini

[9] Maciel, W. J., Costa, R. D. D., Uchida, M. M. M. 2003, A\&A 397, 667

[10] Marigo, P., Bernard-Salas, J., et al., 2003, A\&A 409, 619

[11] Stanghellini, L. 2009, in IAU Symp. 256, The Magellanic Clouds: Stars, Gas, and Galaxies, ed. J. Th. van Loon \& J. M. Oliveira (Dordrecht: Kluwer), 421

[12] Stasińska, G., Richer, M. G., \& McCall, M. 1998, A\&A, 336, 667 